\title{
Erratum
}

\section{Teichmüller curves in moduli space, Eisenstein series and an application to triangular billiards}

\section{W.A. Veech}

Rice University, Department of Mathematics, Box 1892, Houston, TX 77251, USA

Invent. Math. 97, 553-583(1989)

1. " $\operatorname{Re} s>0$ " should $\operatorname{read} " \operatorname{Re} s>\frac{1}{2}$ " (resp. " $\operatorname{Re} s>1$ ") on page 563, lines 13, 10 and page 576, line 3 (resp. p. 563, line 5)

2. "group $(n, n, \infty)$ " should read "group $(2, n, \infty)$ " on page 571 , line 8

3. The parenthetical statement in Theorem 1.1 is a consequence of Proposition 7.8 of the present paper and Theorem 1 of

[16] Takeuchi, K.: Arithmetic triangle groups. J. Math. Soc. Jpn 29, 91-106 (1977)

These corrections do not affect either the statements or the proofs of the results of this paper. 